\section{Paroxysmal Nocturnal Hemoglobinuria: Report of Suboptimal Response to the Use of Eculizumab}

CASE REPORT

\section{Abstract}

Introduction: The Paroxysmal Nocturnal Hemoglobinuria (PNH) is an acquired clonal disorder of one or more stem cells of hematopoietic lineage. It is characterized as a chronic hemolytic anemia in which there is a persistent intravascular hemolysis which is subject to exacerbations due to production of subpopulations of erythrocytes, platelets and granulocytes hypersensitive to complement. Furthermore there has been a high tendency to thrombosis. The anti-complement therapy is the only recognized treatment for this condition. We report a case of PNH with suboptimal response to anti-complement therapy.

Method: This is one case report, obtained through data from medical records of a reference hospital, located in the city of Barbalha, Ceará, Brazil.

Case report: Patient with $\mathrm{PNH}$, with typical clinical features, however nonspecific from the onset of the clinical picture, especially marked by signs and symptoms of anemic syndrome (asthenia, lethargy palpitations, paleness). Showed present hemolysis markers and negative Coombs test. The flow cytometry closed the diagnosis of paroxysmal nocturnal hemoglobinuria and there was the initiation of the anticomplement therapy using the drug called eculizumab. After one year of treatment, the patient persists with anemia and sporadic episodes of hemoglobinuria, as well as present hemolysis markers. However, she remains free of thromboembolic events and without need for transfusion.
Ricardo Parente Garcia Vieira1', Lucas Parente Alencar ${ }^{1}$, George Nilton Nunes Mendes ${ }^{2}$, Natália Parente Alencar2, Francisco Rômulo Patrício de Sá', Hermes Melo Teixeira Batista ${ }^{1,3}$, Jully Graziela Coelho Campos Couto $^{2}$, José Lucas Souza Ramos ${ }^{4}$, Thiáskara Ramile Caldas Leite ${ }^{4}$, Italla Maria Pinheiro Bezerra ${ }^{3}$, Luiz Carlos de Abreu ${ }^{3}$

11 Hospital Regional do Cariri. Juazeiro do Norte-Ce/Brazil

2 Faculty of medicine Juazeiro do Norte-Ce/ Brazil

3 Laboratory of design and Scientific writing of the FMABC. Santo André- São Paulo/ Brazil

4 Faculty of Juazeiro do Norte-Ce/Brazil

Contact information:

\section{Ricardo Parente Garcia Vieira}

巨" ricardo.pgv@gmail.com

\section{Keywords}

paroxysmal nocturnal hemoglobinuria, eculizumab, complement system 
Conclusion: The PNH is a rare pathology with high morbidity and mortality if not treated. The anti-complement therapy with eculizumab offers great chance of long-term control of the most harmful consequences of the disease.

\section{Introduction}

The Paroxysmal Nocturnal Hemoglobinuria (PNH) is an acquired clonal disorder of one or more stem cells of hematopoietic lineage characterized as a chronic hemolytic anemia by persistent intravascular hemolysis and subject to exacerbations due to the production of subpopulations of erythrocytes, platelets and granulocytes hypersensitive to complement. The $\mathrm{PNH}$ is a rare hematologic disorder. The prevalence of 1-1.5 cases per million of residents is estimated. There is no evidence of hereditary susceptibility, occurring in all social classes and both sexes. The diagnosis is usually made around the third to fifth decade of life although it can occur at any age. The most common initial clinical symptoms of the patients with $\mathrm{PNH}$ is characterized by fatigue due to anemia that is present in most cases and can be of varying degrees. The hemoglobinuria is reported as initial presentation in only a minority of the cases. Patients can also refer nausea, abdominal pain, dysphagia, and esophageal spasms resulting from acute intravascular hemolysis outbreaks. The most common physical findings in patients with PNH include paleness and jaundice, the latter being generally mild or even absent.

All patients with an acquired chronic hemolytic anemia with direct negative anti-globulin test (Coombs), particularly if they have hemoglobinuria and patients with myelodysplastic syndromes and aplastic anemia should be evaluated, regardless if there is the presentation of clinically manifest- ed hemolysis, since we can be facing a subclinical $\mathrm{PNH}$. The Flow Cytometry is currently the method of choice for the investigation of $\mathrm{PNH}$, as it is widely available and is able to assess with great sensitivity and specificity the expression of proteins anchored by glycosylphosphatidylinositol (GPI).

The conventional treatment in patients with $\mathrm{PNH}$ is based on hemotherapeutic and clinical support, however in recent years there was great pharmacological breakthrough with the advent of a drug called eculizumab. The degree of hemolysis that these patients are exposed to varies greatly between patients and are related to the size of the $\mathrm{PNH}$ clone and the degree of bone marrow failure: some patients with $\mathrm{PNH}$ present recurrent or persistent episodes of hemolysis and hemoglobinuria, becoming dependent of blood transfusions and may present episodes of thrombosis (especially in the abdominal venous system), main cause of death in individuals with this disease. Others, present rare episodes of hemoglobinuria, controlled hemolytic anemia and rarely require some transfusion support. The only healing treatment in $\mathrm{PNH}$ is the transplantation of hematopoietic stem cells, but this is associated with considerable morbidity and mortality and is indicated only in specific cases.

In this sense, understanding the difficulty of establishing a proper diagnosis because of the low incidence of this pathology, associated with its importance in the correct management of patients with $\mathrm{PNH}$, this study aimed to report a case of $\mathrm{PNH}$ with suboptimal response to the anti-complement therapy. 


\section{Case Report}

Female patient, 37 years old, presenting clinical picture mainly marked by signs and symptoms of the anemic syndrome that started up about one year after the initial treatment (asthenia, lethargy palpitations, pallor) and episodes of dark urine. After the physical examination, we could not observe changes worthy of notification other than moderate mucocutaneous pallor and mild jaundice. Laboratory evaluation revealed anemia $(\mathrm{Hb} 7.8 \mathrm{~g} / \mathrm{dl})$, mild leukopenia, and normal platelet count. There was the presence of levels of lactate dehydrogenase (LDH) increased by about 10 times the upper limit of normal, reticulocytosis(7\%), indirect hyperbilirubinemia with negative direct anti-globulin test (COOMBS). Because the study is about a young patient without comorbidities and with clinical picture marked by acute and recurrent episodes of intravascular hemolysis, the diagnostic possibility of paroxysmal nocturnal hemoglobinuria has been suggested which was confirmed by flow cytometry, which revealed a PNH clone of $45 \%$ in granulocytes. She remained about six months using folic acid and prednisone at low doses and in this period the patient evolved with need of monthly red blood cells transfusion and several episodes of hemoglobinuria. After this period the national public health system released the use of anti-complement therapy with the drug eculizumab. After about 1 year of anti-complement therapy with eculizumab the patient remains free of transfusions and thromboembolic events; however the hemoglobin levels remains between 7-8g/ $\mathrm{dl}$, elevated LDH at about 2 times the upper limit of normal and reticulocytosis.

\section{Discussion}

The present study reports the case of a patient with $\mathrm{PNH}$, presenting typical clinical features, but nonspecific from the onset of the clinical picture, marked especially by signs and symptoms of the anemic syndrome (asthenia, lethargy palpitation, pallor) and the presence of dark urine. The hemoglobinuria, classic sign of $\mathrm{PNH}$ which is present in a minority of the cases, has been reported by the patient. This characteristic finding, however, is present only in the initial report of a minority of patients but when questions are asked directed about the presence of "dark urine," this change would be found more often. [1]

PNH may be present in its classical form in which there is the presence of clinical evidences of intravascular hemolysis without evidence of other manifestations caused by abnormalities in the bone marrow or can occur associated with another specific bone marrow disorder such as bone marrow aplasia, and myelodysplastic syndrome. [2]

Approximately $29-44 \%$ of patients with $\mathrm{PNH}$ have at least one episode of thromboembolism during the evolution of their disease. This complication is also responsible for high mortality rates, accounting for approximately $40-67 \%$ of the causes of death in individuals with such hematological disease. [3]

Thromboembolic phenomena can occur anywhere, but the main sites involved are the supra-hepatic and cerebral veins. Most patients have such phenomena in the first five years after the diagnosis. After three years of clinical follow-up, the patient of the study never showed clinically manifested thromboembolic events. After careful review of the case, it was observed that the patient had positive markers of hemolysis (very high LDH, indirect hyperbilirubinemia, reticulocytosis) and nonreactive direct Coombs. $[3,4,5]$

Due to the presence of hemolytic anemia of unknown cause, there was the decision to investigate the possibility of paroxysmal nocturnal hemoglobinuria. The diagnostic investigation proceeded with the request of a Flow Cytometry (FCM) gold standard test for diagnosis of the PNH. The FCM uses antibodies against the GPI anchored proteins, and the antibodies there are more utilized are the An- 
ti-CD55 and Anti-CD59. The test results showed that the patient had populations of negative granulocytes for CD55 and CD59 which supports and seals the diagnosis of PNH. [6]

The basic treatment for patients with $\mathrm{PNH}$ consisted of hemotherapeutic support, folate replacement and use of corticosteroids, until the approval of the anti-complement therapy. The patient presented repeated episodes of intravascular hemolysis which led to the need of monthly blood transfusions. The only healing treatment consists of the transplant of hematopoietic stem cells. However, this therapeutic modality has considerable morbidity and mortality rates and should only be indicated in selected cases. The eculizumab is a humanized monoclonal antibody directed against the $C 5$ fraction of the terminal complement. It is highly effective in reducing intravascular hemolysis, reducing or even abolishing the need for blood transfusions in patients with $\mathrm{PNH}$ and dramatically reducing the incidence of thromboembolic events. The patient from this case is currently completing one year of use of the drug eculizumab. In this period she remained free of transfusions and had no thromboembolic events. She remains with anemia of mild to moderate intensity and sporadic episodes of hemoglobinuria. Though admittedly effective, the high cost is a limiting factor for the use of Eculizumab in developing countries. $[6,7]$

\section{Conclusion}

The PNH is a rare pathology with high morbidity and mortality if not treated. The anti-complement therapy with eculizumab offers great chance of long-term control of the most harmful consequences of the disease (thromboembolic events, need for transfusion). We report a case of suboptimal response to eculizumab (persistence of anemia and hemoglobinuria crisis). However, the same therapy obtained great success in controlling the transfusion need and the occurrence of thromboembolic events. 


\section{References}

1. Luzzato, L, Notaro R. Paroxysmal Nocturnal Haemoglobinuria. In: Principles and practice of hematology. Handin, R.I.; Lux, S.E.; Stosser, T.P. 2 ed, Lippincott Williams 8 Wilkins, 2003-2004. Chapter 10, p. 328-32.

2. De Latour RP, et al. Paroxysmal nocturnal hemoglobinuria: natural history of disease subcategories. Blood. 2008; 112(8): 3099-3106

3. Hill A, Kelly RJ, Hillmen P. Thrombosis in paroxysmal nocturnal hemoglobinuria. Blood. 2013; 121(25): 4985 - 4996

4. Arruda MMA, et al. Hemoglobinúria paroxística noturna: da fisiopatologia ao tratamento. Rev Assoc Med Bras. 2010; 56: 214-21

5. Brodsky RA. Paroxysmal nocturnal hemoglobinuria. Blood. 2014; 124(18): $2804-281$

6. Parker $C$, et al. Diagnosis and management of paroxysmal nocturnal hemoglobinúria. Blood. 2005; 106(12): 3699 - 3709

7. Brodsky RA. How I treat paroxysmal nocturnal hemoglobinúria. Blood. 2009; 113(26): 6522 - 6527

8. Mahmood A, Sharif MA, Murtaza B. . Paroxysmal Nocturnal Hemoglobinuria. Journal of the Collage of Physicians and Surgeons Pakistan. 2008; 18(8): 512-14

9. Modesto TM, et al. Importância e vantagem da citometria de fluxo frente aos testes de triagem no diagnostico da Hemoglobinúria Paroxística Noturna. Rev. Bras. Hematol. Hemoter. 2006; 28(4) 275-279

10. Nomura $\mathrm{ML}$, et al. Hemoglobinúria paroxística noturna e gravidez. RBGO. 2004; 26(7):579-82

\section{Comment on this article:}
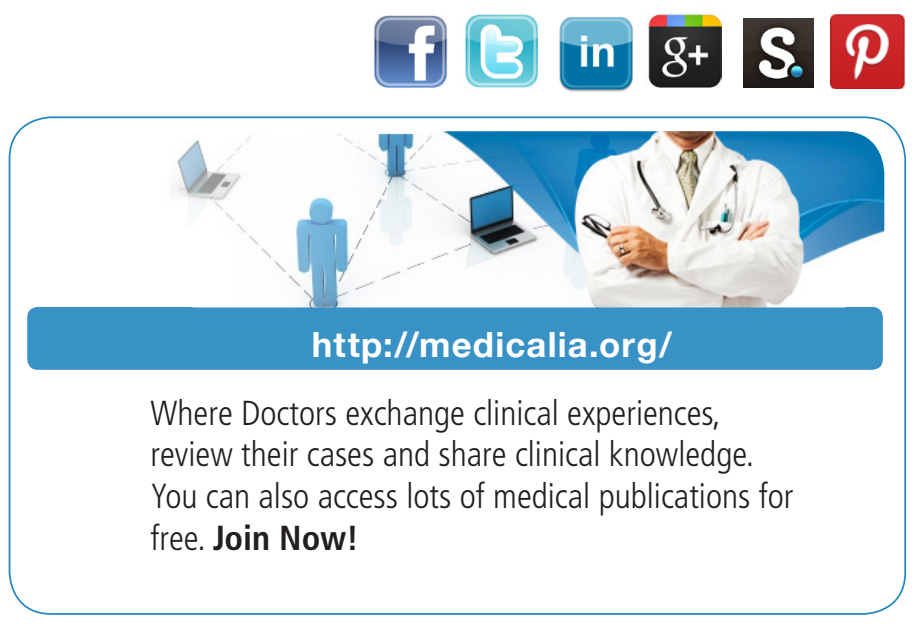

\section{Publish with iMedPub}

\section{http://www.imed.pub}

International Archives of Medicine is an open access journal publishing articles encompassing all aspects of medical science and clinical practice. IAM is considered a megajournal with independent sections on all areas of medicine. IAM is a really international journal with authors and board members from all around the world. The journal is widely indexed and classified Q1 in category Medicine. 